\title{
Conjugate Effects of Radiation Flux on Double Diffusive MHD Free Convection Flow of a Nanofluid over a Power Law Stretching Sheet
}

\author{
Muhammad Imran Anwar, ${ }^{1}$ Sharidan Shafie, ${ }^{1}$ Ilyas Khan, ${ }^{1}$ and Mohd Zuki Salleh ${ }^{2}$ \\ ${ }^{1}$ Department of Mathematical Sciences, Faculty of Science, Universiti Teknologi Malaysia (UTM), Johor, 81310 Skudai, Malaysia \\ ${ }^{2}$ Faculty of Industrial Science and Technology, University Malaysia Pahang (UMP), Pahang, 26300 Kuantan, Malaysia
}

Correspondence should be addressed to Sharidan Shafie, ridafie@yahoo.com

Received 17 September 2012; Accepted 30 October 2012

Academic Editors: A. Ghoufi, R. D. Simitev, and Z. Xu

Copyright (c) 2012 Muhammad Imran Anwar et al. This is an open access article distributed under the Creative Commons Attribution License, which permits unrestricted use, distribution, and reproduction in any medium, provided the original work is properly cited.

\begin{abstract}
This study theoretically investigates the conjugate effects of radiation flux and magnetohydrodynamic (MHD) on free convection boundary layer flow of a nanofluid over a nonlinear stretching sheet. It is assumed that the magnetic Reynolds number is small enough and the sheet is stretched with a power law velocity under the effects of the magnetic field, the buoyancy parameter, and the solutal buoyancy parameter. The model used for the nanofluid incorporates the effects of Rosseland approximation, Brownian motion, and thermophoresis parameters. By using appropriate similarity transformations, the governing nonlinear partial differential equations are transformed into dimensionless form and numerically solved using an implicit finite difference scheme known as the Keller-box method. It is found that the variations of magnetic field, buoyancy parameter, solutal buoyancy parameter, and the power law velocity parameter have strong influence on the motion.
\end{abstract}

\section{Introduction}

Heat and mass transfer (double-diffusion) phenomenon on free convection is driven by two density gradients which have different rates of diffusion and currently is an important fluid dynamics topic. A common example of double diffusive convection appears in oceanography, where heat and salt concentrations exist with different gradients and diffuse at differing rates. Double diffusive convection is also important in understanding the evolution of a number of systems that have multiple causes for density variations. These include convection in the earth's oceans, in magma chambers, and in the sun where heat and helium diffuse at differing rates [1]. Double diffusive convection flows for Newtonian and non-Newtonian fluids are extensively studied. However, for nanofluids such studies are scarce due to their complicated nature $[2,3]$. Moreover, recent developments in the field of fluid dynamics and nanotechnology confirm that nanofluids are industrially more important than other available fluids [4]. There are numerous biomedical applications that involve nanofluids such as magnetic cell separation, drug delivery, hyperthermia, and contrast enhancement in magnetic resonance imaging [5]. Hence, this is the motivation for considering nanofluids in the present work.

On the other hand, stretching sheet problems with double diffusion are important in extrusion process, glass fibber, paper production, hot rolling, wire drawing, electronic chips, crystal growing, plastic manufactures, food processing, and movement of biological fluids [6]. Khan and Pop [7] investigated the laminar flow of a nanofluid on a stretching flat surface by incorporating the effects of Brownian motion, thermophoresis and reported to be the pioneer for this study of stretching sheet in nanofluid. Rana and Bhargava [8] discussed the flow and heat transfer of a nanofluid over a nonlinearly stretching sheet. Furthermore, the MHD flow of nanofluid over a power law stretching sheet plays an important role in various industrial applications including magnetic control of molten iron flow in the steel industry and liquid metal cooling in nuclear reactors [9]. In addition, when thermal radiation is considered, such studies have useful chemical processing applications $[10,11]$. 
The aim of the present work is to numerically investigate the thermal radiation effects for double diffusive MHD free convection flow of a nanofluid over a power law stretching sheet using Keller-box method [12, 13].

\section{Problem Formulation}

The unsteady two-dimensional boundary layer flow of a nanofluid past a stretching surface is considered. The stretching velocity is assumed to be $u_{w}(x)=a x^{m}$, where $a$ is constant, $m(m \geq 0)$ is the power law exponent parameter, and $x$ is the coordinate measured along the stretching surface. The flow takes place above the stretching surface at $y \geq$ 0 . Here $y$ is the coordinate axis measured normal to the stretching surface. A uniform stress leading to equal and opposite forces is applied along the $x$-axis, so that the sheet is stretched keeping the origin fixed. Following the work of Hayat and Qasim [6] and Afify [14], we assume that a uniform transverse magnetic field of strength $B$ is imposed parallel to the $y$-axis and the induced magnetic field due to the motion of the electrically-conducting fluid is negligible. We also assume that the external electrical field is zero and the electric field due to the polarization of charges is negligible. Moreover, it is further supposed that at the stretching surface, the temperature $T$ and the nanoparticle fraction $C$ take constant values $T_{w}$ and $C_{w}$, whereas the ambient values of temperature $T_{\infty}$ and the nanoparticle fraction $C_{\infty}$ are attained as $y$ tends to infinity. The Oberbeck-Boussinesq approximation is employed to the field equations. The governing boundary layer equations that are based on the balance laws of mass, linear momentum, energy, and concentration species for the present problem are given as follows $[3,15]$ :

$$
\begin{gathered}
\nabla \cdot \mathbf{V}=0 \\
\rho_{f}\left(\frac{\partial \mathbf{V}}{\partial t}+(\mathbf{V} \cdot \nabla) \mathbf{V}\right) \\
=-\nabla p+\mu \nabla^{2} \mathbf{V}-\sigma B^{2}(x) \mathbf{V} \\
+\left[\left(1-C_{\infty}\right) \rho_{f_{\infty}} \beta_{T}\left(T-T_{\infty}\right)+\left(\rho_{p}-\rho_{f_{\infty}}\right) \beta_{C}\left(C-C_{\infty}\right)\right] \mathbf{g}, \\
(\rho c)_{f}\left(\frac{\partial T}{\partial t}+\mathbf{V} \cdot \nabla T\right) \\
=k \nabla^{2} T+(\rho c)_{p}\left[D_{B} \nabla C \cdot \nabla T+\left(\frac{D_{T}}{T_{\infty}}\right) \nabla T \cdot \nabla T\right] \\
-\nabla \cdot q_{r}, \quad \frac{\partial C}{\partial t}+\mathbf{V} \cdot \nabla C=D_{B} \nabla C+\left(\frac{D_{T}}{T_{\infty}}\right) \nabla^{2} T,
\end{gathered}
$$

where $\mathbf{V}$ is the velocity vector, $\sigma$ is the electrical conductivity, $\mathrm{g}$ is the acceleration due to gravity, $\mu$ is the viscosity, $\rho_{f}$ is the density of the base fluid, $\rho_{p}$ is the density of the nanoparticle, $\beta_{T}$ is the coefficient of volumetric thermal expansion, $\beta_{C}$ is the coefficient of volumetric concentration expansion, $D_{B}$ is the Brownian diffusion coefficient, $D_{T}$ is the thermophoretic diffusion coefficient, $k$ is the thermal conductivity, $(\rho c)_{p}$ is the heat capacitance of the nanoparticles, $(\rho c)_{f}$ is the heat capacitance of the base fluid, and $q_{r}$ is the radiation flux.

In the following we adopt the Rosseland approximation

$$
q_{r}=-\frac{4 \sigma^{*}}{3 k^{*}} \frac{\partial T^{4}}{\partial y}
$$

where $\sigma^{*}$ is the Stefan-Boltzmann constant and $k^{*}$ is the mean absorption coefficient. Assuming that the temperature difference between the free stream $T_{\infty}$ and the fluid temperature $T$ is small enough, expanding $T^{4}$ in a Taylor series about $T_{\infty}$, and neglecting higher order terms, it results that

$$
T^{4}=4 T_{\infty}^{3} T-3 T_{\infty}^{4} .
$$

The two-dimensional steady-state problem under the assumptions that the external pressure in $x$-direction having diluted nanoparticles is constant; that is, $p=p_{0}$ yields to $\partial p / \partial x=0$ and Oberbeck-Boussinesq approximation is valid; (1) are reduced to

$$
\begin{gathered}
\frac{\partial u}{\partial x}+\frac{\partial v}{\partial y}=0, \\
0=\mu \frac{\partial^{2} u}{\partial y^{2}}-\rho_{f}\left(u \frac{\partial u}{\partial x}+v \frac{\partial u}{\partial y}\right)-\sigma B^{2}(x) u \\
+\left[\left(1-C_{\infty}\right) \rho_{f_{\infty}} g \beta_{T}\left(T-T_{\infty}\right)\right. \\
\left.-\left(\rho_{p}-\rho_{f_{\infty}}\right) g \beta_{C}\left(C-C_{\infty}\right)\right], \\
u \frac{\partial T}{\partial x}+v \frac{\partial T}{\partial y} \\
=\alpha \nabla^{2} T+\tau\left[D_{B} \frac{\partial C}{\partial y} \frac{\partial T}{\partial y}+\frac{D_{T}}{T_{\infty}}\left(\frac{\partial T}{\partial y}\right)^{2}\right] \\
-\frac{1}{(\rho c)_{f}} \frac{\partial q_{r}}{\partial y}, \\
u \frac{\partial C}{\partial x}+v \frac{\partial C}{\partial y}=D_{B} \frac{\partial^{2} C}{\partial y^{2}}+\frac{D_{T}}{T_{\infty}} \frac{\partial^{2} T}{\partial y^{2}},
\end{gathered}
$$

where

$$
\alpha=\frac{k}{(\rho c)_{f}}, \quad \tau=\frac{(\rho c)_{p}}{(\rho c)_{f}} .
$$

Here $u$ and $v$ are the velocity components in the $x$ and $y$ directions, respectively, $\alpha$ is the thermal diffusivity parameter and $\tau$ is the ratio between the effective heat capacity of the nanoparticle material and heat capacity of the fluid.

The associated boundary conditions are

$$
\begin{array}{r}
u=u_{w}(x)=a x^{m}, \quad v=0, \quad T=T_{w}, \quad C=C_{w} \\
\text { at } y=0, \\
u \longrightarrow 0, \quad v \longrightarrow 0, \quad T \longrightarrow T_{\infty}, \quad C \longrightarrow C_{\infty} \\
\text { as } y \longrightarrow \infty .
\end{array}
$$


Using the stream function $\psi=\psi(x, y)$, the components of velocity are defined as

$$
u=\frac{\partial \psi}{\partial y}, \quad v=-\frac{\partial \psi}{\partial x} .
$$

By using the following similarity transformations [14],

$$
\begin{aligned}
& \psi=\sqrt{\frac{2 \operatorname{vax}^{m+1}}{m+1}} f(\eta), \quad \theta(\eta)=\frac{T-T_{\infty}}{T_{w}-T_{\infty}}, \\
& \phi(\eta)=\frac{C-C_{\infty}}{C_{w}-C_{\infty}}, \quad \eta=y \sqrt{\frac{(m+1) a x^{m-1}}{2 v}},
\end{aligned}
$$

into (4), we get

$$
\begin{gathered}
f^{\prime \prime \prime}+f f^{\prime \prime}-\frac{2 m}{m+1} f^{\prime 2}+\frac{2}{m+1}\left(M^{2} f^{\prime}+\lambda \theta-\delta \phi\right)=0 \\
\frac{1}{\operatorname{Pr}}(1+N) \theta^{\prime \prime}+f \theta^{\prime}+N b \phi^{\prime} \theta^{\prime}+N t \theta^{\prime 2}=0 \\
\phi^{\prime \prime}+\operatorname{Le} f \phi^{\prime}+\frac{N t}{N b} \theta^{\prime \prime}=0
\end{gathered}
$$

where

$$
\begin{gathered}
M^{2}=\frac{\sigma B_{0}^{2}}{a \rho_{f}}, \quad \lambda=\frac{G r}{\operatorname{Re}_{x}^{3 / 2}}, \quad \delta=\frac{G m}{\operatorname{Re}_{x}^{3 / 2}}, \\
\operatorname{Pr}=\frac{\nu}{\alpha}, \quad N=\frac{16 \sigma^{*} T_{\infty}^{3}}{3 k k^{*}}, \quad \mathrm{Le}=\frac{\nu}{D_{B}}, \\
\nu=\frac{\mu}{\rho_{f}}, \quad N b=\frac{\tau D_{B}\left(C_{w}-C_{\infty}\right)}{\nu}, \\
N t=\frac{\tau D_{T}\left(T_{w}-T_{\infty}\right)}{\nu T_{\infty}}, \quad \operatorname{Re}_{x}=\frac{u_{w}(x) x}{\nu}, \\
G r=\frac{\left(1-C_{\infty}\right)\left(\rho_{f_{\infty}} / \rho_{f}\right) g n\left(T_{w}-T_{\infty}\right)}{\nu^{2} \operatorname{Re}_{x}^{1 / 2}}, \\
G m=\frac{\left(\left(\rho_{p}-\rho_{f_{\infty}}\right) / \rho_{f}\right) g n_{1}\left(C_{w}-C_{\infty}\right)}{v^{2} \operatorname{Re}_{x}^{1 / 2}} .
\end{gathered}
$$

Here $M$ is the magnetic parameter called Hartmann number, $\lambda$ is the buoyancy parameter, $\delta$ is the solutal buoyancy parameter, $\operatorname{Pr}$ is the Prandtl number, $N$ is the radiation parameter, Le is the Lewis number, $v$ is the kinematic viscosity of the fluid, $\mathrm{Nb}$ is the Brownian motion parameter, $N t$ is the thermophoresis parameter, $\mathrm{Re}_{x}$ is the local Reynolds number based on the stretching velocity, $G r$ is the local thermal Grashof number, $G m$ is the local concentration Grashof number, and $f, \theta$, and $\phi$ are the dimensionless stream functions, temperature, and rescaled nanoparticle volume fraction, respectively. Further, in order to eliminate the dependence of $M$ on $x$, the magnetic field strength has been taken proportional to $x$, that is, $B(x)=$ $B_{0} x^{(m-1) / 2}$ where $B_{0}$ is a constant [14]. Also, $\beta_{T}$ and $\beta_{C}$ are proportional to $x^{-3}$, that is, $\beta_{T}=n x^{-3}, \beta_{C}=n_{1} x^{-3}$, where $n$ and $n_{1}$ are constants of proportionality [16].
The corresponding boundary conditions are transformed to

$$
\begin{gathered}
f=0, \quad f^{\prime}=1, \quad \theta=1, \quad \phi=1 \\
\text { at } \eta=0, \\
f^{\prime} \longrightarrow 0, \quad \theta \longrightarrow 0, \quad \phi \longrightarrow 0 \quad \text { as } \eta \longrightarrow \infty .
\end{gathered}
$$

In the present case, we define Nusselt number, Sherwood number, and skin-friction as follows:

$$
\begin{gathered}
\mathrm{Nu}=\frac{q_{w} x}{k\left(T_{w}-T_{\infty}\right)}, \quad \mathrm{Sh}=\frac{q_{m} x}{D_{B}\left(C_{w}-C_{\infty}\right)}, \\
C_{f}=\frac{\tau_{w}}{(1 / 2) \rho U^{2}},
\end{gathered}
$$

where

$$
\begin{array}{r}
q_{w}=-k \frac{\partial T}{\partial y}, \quad q_{m}=-D_{B} \frac{\partial C}{\partial y}, \quad \tau_{w}=\mu \frac{\partial u}{\partial y}, \\
\text { at } y=0 .
\end{array}
$$

The associated expressions of the dimensionless reduced Nusselt number $-\theta^{\prime}(0)$, reduced Sherwood number $-\phi^{\prime}(0)$, and skin-friction coefficient $C_{f x}$ are defined as

$$
\begin{gathered}
\mathrm{Nu}_{r}=\frac{\mathrm{Nu}}{\sqrt{((m+1) / 2) \mathrm{Re}_{x}}}, \quad \mathrm{Sh}_{r}=\frac{\mathrm{Sh}}{\sqrt{((m+1) / 2) \mathrm{Re}_{x}}}, \\
C_{f x}=\frac{C_{f}}{2} \sqrt{\frac{2}{m+1} \operatorname{Re}_{x} .}
\end{gathered}
$$

The transformed nonlinear ordinary differential (9) with the boundary conditions (11) are numerically solved by means of the Keller-box method $[12,13]$.

\section{Results and Discussion}

Numerical results for some physical parameters of interest are shown in Tables 1 and 2 and in Figures 1-9. In Table 1, the results for reduced Nusselt number $-\theta^{\prime}(0)$ and reduced Sherwood number $-\phi^{\prime}(0)$, when the Hartmann number $M$, the buoyancy parameter $\lambda$, the solutal buoyancy parameter $\delta$, and the radiation parameter $N$ are zero and the power law velocity parameter $m=1$, are compared with those obtained by Khan and Pop [7]. We found that, this comparison shows an excellent agreement for the involved flow parameters and in general their solutions appeared as the special cases of our general solutions. Similarly if we take $\mathrm{Nb}=0.5, \mathrm{Nt}=$ $0.5, \operatorname{Pr}=2.0, \mathrm{Le}=2.0$, and $m=2.0$, the results of Rana and Bhargava [8] can also be obtained as a special case. Hence we are quite confident that our results are accurate and are more general than the existing studies in the literature.

The variations of reduced Nusselt number $-\theta^{\prime}(0)$, reduced Sherwood number $-\phi^{\prime}(0)$, and the skin-friction coefficient $C_{f x}$ for different values of $N b, N t$, Pr, Le, $M, \lambda$, $\delta, N$, and $m$ are shown in Table 2 . It is observed that $-\theta^{\prime}(0)$ is a decreasing function of $N b, N t, M, \delta, N$, and $m$, whereas $-\theta^{\prime}(0)$ increases with increasing values of $\operatorname{Pr}$, Le, and $\lambda$. 
TABLE 1: Comparison of reduced Nusselt number and reduced Sherwood number.

\begin{tabular}{|c|c|c|c|c|c|c|c|c|c|c|c|c|}
\hline \multirow{2}{*}{$\mathrm{Nb}$} & \multirow{2}{*}{$N t$} & \multirow{2}{*}{$\operatorname{Pr}$} & \multirow{2}{*}{ Le } & \multirow{2}{*}{$M$} & \multirow{2}{*}{$\lambda$} & \multirow{2}{*}{$\delta$} & \multirow{2}{*}{$N$} & \multirow{2}{*}{$m$} & \multicolumn{2}{|c|}{ Khan and Pop [7] } & \multicolumn{2}{|c|}{ Present result } \\
\hline & & & & & & & & & $-\theta^{\prime}(0)$ & $-\phi^{\prime}(0)$ & $-\theta^{\prime}(0)$ & $-\phi^{\prime}(0)$ \\
\hline 0.1 & 0.1 & 10 & 10 & 0.0 & 0.0 & 0.0 & 0.0 & 1.0 & 0.9524 & 2.1294 & 0.9524 & 2.1294 \\
\hline 0.2 & 0.2 & 10 & 10 & 0.0 & 0.0 & 0.0 & 0.0 & 1.0 & 0.3654 & 2.5152 & 0.3654 & 2.5152 \\
\hline 0.3 & 0.3 & 10 & 10 & 0.0 & 0.0 & 0.0 & 0.0 & 1.0 & 0.1355 & 2.6088 & 0.1355 & 2.6088 \\
\hline 0.4 & 0.4 & 10 & 10 & 0.0 & 0.0 & 0.0 & 0.0 & 1.0 & 0.0495 & 2.6038 & 0.0495 & 2.6038 \\
\hline 0.5 & 0.5 & 10 & 10 & 0.0 & 0.0 & 0.0 & 0.0 & 1.0 & 0.0179 & 2.5731 & 0.0179 & 2.5731 \\
\hline
\end{tabular}

TABLE 2: Variations of reduced Nusselt number, reduced Sherwood number, and skin-friction coefficient.

\begin{tabular}{cccccccccccc}
\hline$N b$ & $N t$ & $\operatorname{Pr}$ & Le & $M$ & $\lambda$ & $\delta$ & $N$ & $m$ & $-\theta^{\prime}(0)$ & $-\phi^{\prime}(0)$ & $C_{f x}$ \\
\hline 0.1 & 0.1 & 0.71 & 10 & 0.1 & 1.0 & 1.0 & 1.0 & 0.5 & 0.3399 & 2.3369 \\
$\mathbf{0 . 5}$ & 0.1 & 0.71 & 10 & 0.1 & 1.0 & 1.0 & 1.0 & 0.5 & 0.3004 & 2.3976 \\
0.1 & $\mathbf{0 . 5}$ & 0.71 & 10 & 0.1 & 1.0 & 1.0 & 1.0 & 0.5 & 0.3131 & 2.1134 & 0.6148 \\
0.1 & 0.1 & $\mathbf{7 . 0 0}$ & 10 & 0.1 & 1.0 & 1.0 & 1.0 & 0.5 & 0.8824 & 2.0780 & 0.6473 \\
0.1 & 0.1 & 0.71 & $\mathbf{2 5}$ & 0.1 & 1.0 & 1.0 & 1.0 & 0.5 & 0.3418 & 3.8356 & 0.5670 \\
0.1 & 0.1 & 0.71 & 10 & $\mathbf{2 . 0}$ & 1.0 & 1.0 & 1.0 & 0.5 & 0.2393 & 2.1222 & 1.6688 \\
0.1 & 0.1 & 0.71 & 10 & 0.1 & $\mathbf{3 . 0}$ & 1.0 & 1.0 & 0.5 & 0.4042 & 2.4740 & -0.1679 \\
0.1 & 0.1 & 0.71 & 10 & 0.1 & 1.0 & $\mathbf{4 . 0}$ & 1.0 & 0.5 & 0.3265 & 2.2761 & 1.0759 \\
0.1 & 0.1 & 0.71 & 10 & 0.1 & 1.0 & 1.0 & $\mathbf{6 . 0}$ & 0.5 & 0.1817 & 2.4120 & 0.4856 \\
0.1 & 0.1 & 0.71 & 10 & 0.1 & 1.0 & 1.0 & 1.0 & $\mathbf{5 . 0}$ & 0.2804 & 2.2294 & 1.1273 \\
\hline
\end{tabular}

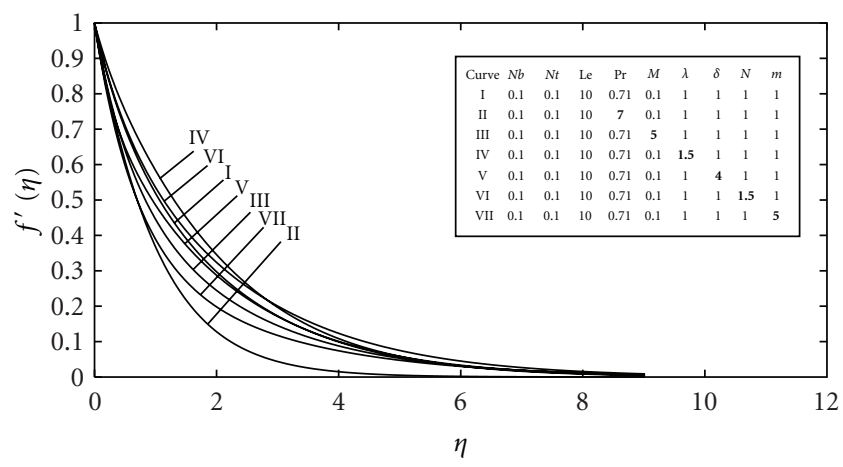

FIGURE 1: Variations of velocity profiles with $\eta$ for different values of $\operatorname{Pr}, M, \lambda, \delta, N$, and $m$.

However, it is found that $-\phi^{\prime}(0)$ decreases for large values of $N t, \operatorname{Pr}, M, \delta$, and $m$ and increases for increasing values of $N b$, Le, $\lambda$, and $N$. It is further observed from this table that $C_{f x}$ is an increasing function of $N t, \operatorname{Pr}, M, \delta$, and $m$ and a decreasing one with respect to $N b$, Le, $\lambda$, and $N$.

In order to get physical insight into the problem, convergence of plots for dimensionless velocity, temperature and concentration profiles for different flow parameters are provided in Figures 1-3. Furthermore, the variations of the reduced Nusselt number, the reduced Sherwood number, and skin-friction coefficient as a function of $\lambda$ and $\delta$ are shown in Figures 4-9. All these graphs are plotted for fixed values of $N b=N t=0.1$ and Le $=10$.

It is evident from Figure 1 that an increase in Pr results in a decrease in the velocity, whereas velocity increases for the increasing values of $\lambda$ and $N$. The effect of increasing values of $M$ results in a decrease of momentum boundary layer

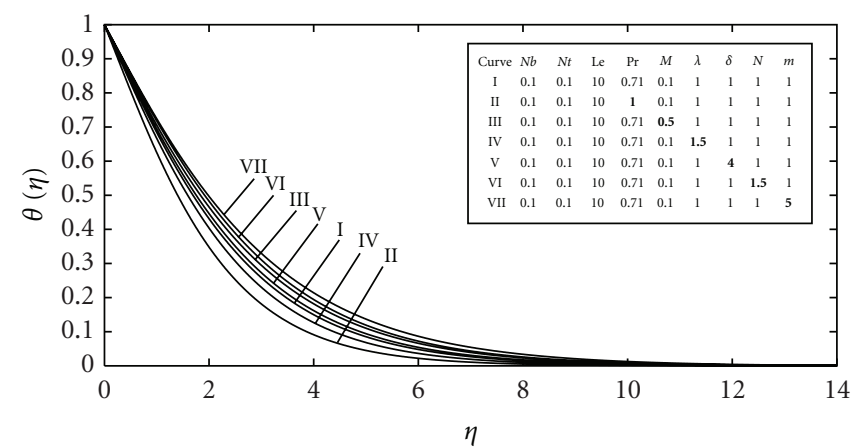

FIGURE 2: Variations of temperature profiles with $\eta$ for different values of $\operatorname{Pr}, M, \lambda, \delta, N$, and $m$.

thickness which reduces the dimensionless stream function and the velocity of nanofluid. Physically, it is possible due to the fact that an increase in Pr makes the fluid to be more viscous, which leads to decrease in the velocity for increasing values of Hartmann number and increase the frictional force which reduce the fluid velocity to slow down the fluid motion. Further, it is found that velocity decreases with increasing values of $\delta$ and $m$.

Figure 2 is plotted to analyze the heat transfer for combined effects of $\operatorname{Pr}, M, \lambda, \delta, N$, and $m$ on the temperature profiles. This figure indicates that an increase in Pr and $\lambda$ results in a decrease in the temperature profiles, whereas these profiles increase for the increasing values of $\delta, N$, and $m$. The radiation parameter $N$ is very effective for the thickening of the thermal boundary layer which releases the heat energy from the flow region and causes the cooling of the system. Physically, it is true due to the fact that temperature increases by increasing the Rosseland approximation. 


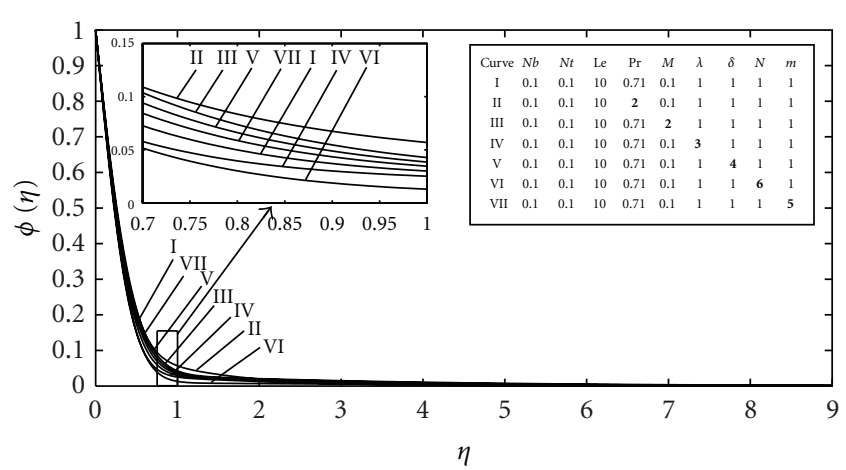

Figure 3: Variations of concentration profiles with $\eta$ for different values of $\operatorname{Pr}, M, \lambda, \delta, N$, and $m$.

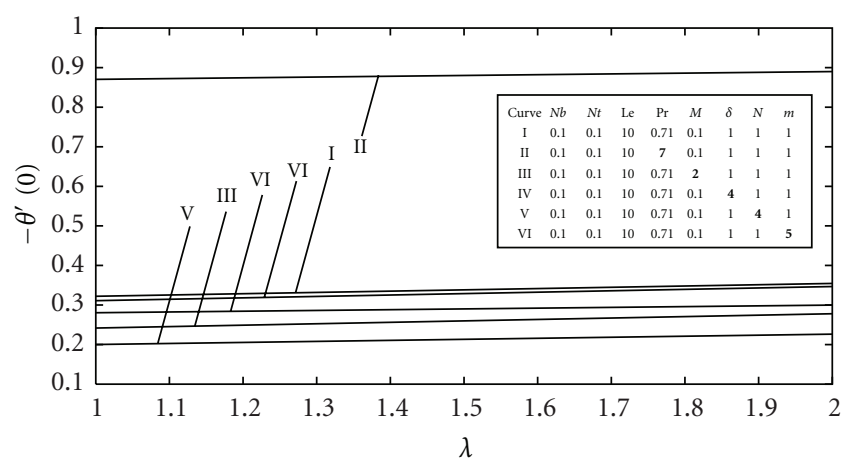

FIGURE 4: Variations of reduced Nusselt number with $\lambda$ for different values of $\operatorname{Pr}, M, \lambda, \delta, N$, and $m$.

Increasing values of $M$ cause the temperature to rise up. This is due to the fact that nanoparticles dissipate energy in the form of heat which causes the thermal boundary layer thickness to increase in the case of nanofluids and ultimately a localized rise in temperature of the fluid occurs.

Figure 3 is prepared to study the effects of incorporated different flow parameters on concentration profiles. It results that the increasing values of $\lambda$ and $N$ cause a decrease in the mass transfer. However, the concentration increases for large values of $\operatorname{Pr}, M, \delta$, and $m$. Physically, the variations of $\operatorname{Pr}, M$, $\lambda, \delta$, and $m$ are important in the boundary layer flow and play a significant role in shortening the concentration boundary layer for the mass fraction. All profiles discussed above descend smoothly in the free stream satisfying boundary conditions. This ensures the accuracy of the obtained numerical results.

The variations of the reduced Nusselt number, reduced Sherwood number, and skin-fiction coefficient as functions of $\lambda$ for the different values of $\operatorname{Pr}, M, \delta, N$, and $m$ are shown in Figures 4-6. From Figure 4, we observed that the reduced Nusselt number increases for increasing values of $\operatorname{Pr}$ and decreases for increasing values of $M, \delta, N$, and $m$. This increase in the heat transfer rate at the surface of the sheet is due to the reason that nanofluids with high viscosities have large values of Prandtl number which results for relatively low thermal conductivities to reduce

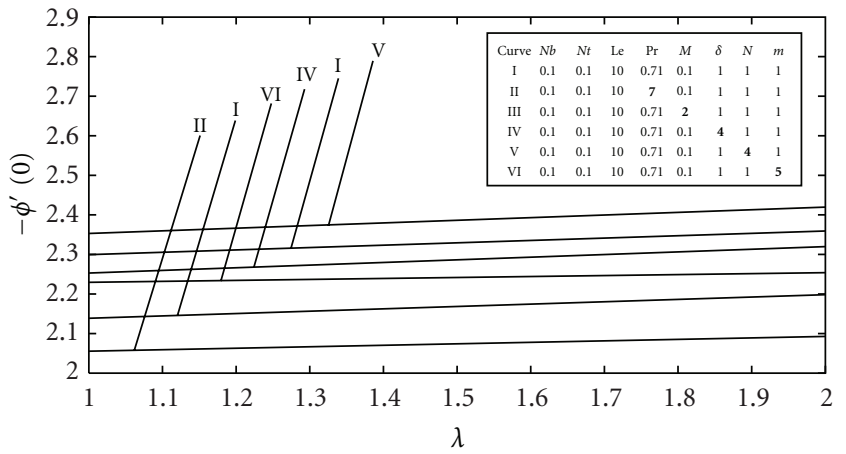

FIGURE 5: Variations of reduced Sherwood number with $\lambda$ for different values of $\operatorname{Pr}, M, \lambda, \delta, N$, and $m$.

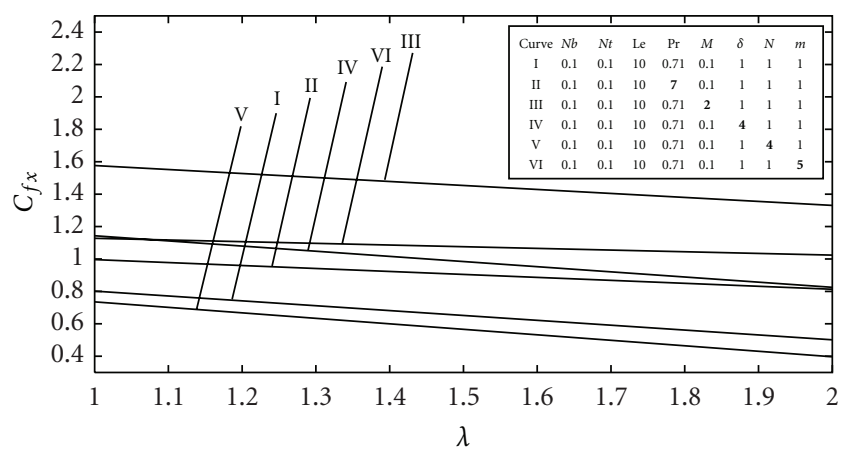

Figure 6: Variations of skin-friction coefficient with $\lambda$ for different values of $\operatorname{Pr}, M, \lambda, \delta, N$, and $m$.

the conduction phenomenon [7]. Physically, this behaviour is meaningful due to the fact that with increasing viscosity of the base fluid, the thermal boundary layer thickness decreases because of less colloidal forces between the base fluid and suspended nanoparticles. So the thermal conductivities of nanofluids play an important role to increase the heat transfer coefficient between the heat transfer medium and heat transfer surface. Further, Figure 5 shows that the reduced Sherwood number decreases for increasing values of Pr but increases for increasing values of $N, M, \delta$, and $m$. It is clearly seen from Figure 6 that the dimensionless skinfriction coefficient along $\lambda$ increases with increasing values of $\operatorname{Pr}, M, \delta$, and $m$ but decreases with increasing values of $N$.

Finally, Figures 7-9 show the variation in the reduced Nusselt number, the reduced Sherwood number, and skinfiction coefficient along $\delta$ for different values of $\operatorname{Pr}, M, \lambda, N$, and $m$. It is found from Figure 7 that the reduced Nusselt number decreases for increasing values of $M, N$, and $m$ and increases for increasing values of $\operatorname{Pr}$ and $\lambda$. The reduced Sherwood number increases for large values of $\lambda$ and $N$ but decreases when the values of $\operatorname{Pr}, M$, and $m$ are increased as shown in Figure 8. Further, Figure 9 shows that skin-friction coefficient increases for the larger values of $\operatorname{Pr}, M$, and $m$ and decreases with regard to $\lambda$ and $N$. It is clearly seen that the influence of these parameters on the local Sherwood number and skin-fiction coefficient with $\lambda$ and $\delta$ is quite opposite to each other. 


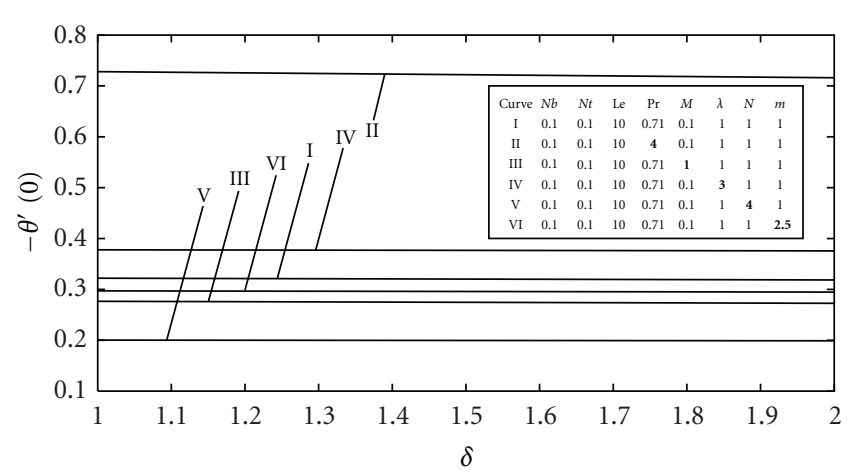

FIgURE 7: Variations of reduced Nusselt number with $\delta$ for different values of $\operatorname{Pr}, M, \lambda, \delta, N$, and $m$.

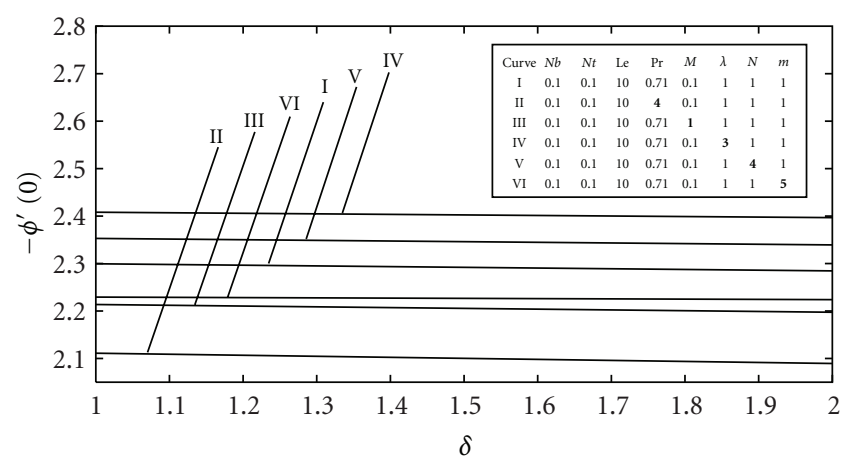

Figure 8: Variations of reduced Sherwood number with $\delta$ for different values of $\operatorname{Pr}, M, \lambda, \delta, N$, and $m$.

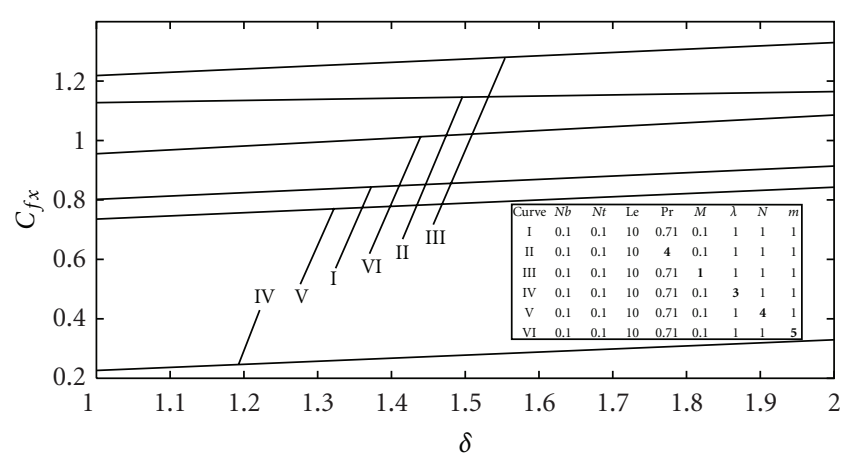

FIGURE 9: Variations of skin-friction coefficient with $\delta$ for different values of $\operatorname{Pr}, M, \lambda, \delta, N$, and $m$.

\section{Conclsions}

In the present study we have investigated the conjugate effects of thermal radiation flux on double diffusive MHD free convection boundary layer flow of a nanofluid over a nonlinear stretching sheet. The model used here for nanofluid incorporates the effects of thermophoresis parameter $\mathrm{Nb}$, Brownian motion parameter $N t$, Prandtl number Pr, Lewis number Le, Hartmann number $M$, buoyancy parameter $\lambda$, solutal buoyancy parameter $\delta$, radiation parameter $N$, and nonlinear stretching parameter $m$. The governing nonlinear partial differential equations are numerically solved using implicit finite difference scheme known as Keller-box method. Numerical solutions depending upon all parameters are presented in tables and figures. The physical interpretations provided by the authors reflect some important characteristics of free convection flow of a nanofluid over a power law stretching sheet and play a significant role in the concentration boundary layer for the mass fraction. The following main results are concluded from this study.

(1) The reduced Nusselt number $-\theta^{\prime}(0)$ is a decreasing function of $N b, N t, M, \delta, N$, and $m$ whereas increases with increasing values of $\mathrm{Pr}, \mathrm{Le}$, and $\lambda$.

(2) The reduced Sherwood number $-\phi^{\prime}(0)$ decreases for large values of $N t, \operatorname{Pr}, M, \delta$, and $m$ and increases for increasing values of $N b$, Le, $\lambda$, and $N$.

(3) The skin-friction coefficient $C_{f x}$ is an increasing function of $N t, \operatorname{Pr}, M, \delta$, and $m$ and a decreasing one with respect to $N b$, Le, $\lambda$, and $N$.

(4) The velocity of nanofluid decreases for increasing $\operatorname{Pr}$ whereas increases for the increasing values of $\lambda$ and $N$.

(5) The temperature of nanofluid decreases when Pr and $\lambda$ are increased whereas increases for large values of $M, \delta$, and $m$.

(6) The species concentration of nanofluid decreases for large values of $\lambda$ and $N$ whereas increases for increasing values of $\operatorname{Pr}, M, \delta$, and $m$.

(7) The reduced Nusselt number versus $\lambda$ increases for Pr and decreases for $M, \delta, N$, and $m$ whereas the reduced Nusselt number against $\delta$ decreases for $M$, $N$, and $m$ while increases for $\operatorname{Pr}$ and $\lambda$. However, the reduced Sherwood number and skin-friction coefficient versus $\lambda$ and $\delta$ are found to be opposite in nature.

\section{Acknowledgments}

The authors would like to acknowledge the financial support received from MOHE, Research Management Centre-RMC (4F109), UTM, Malaysia, and University Malaysia Pahang (RDU110108).

\section{References}

[1] W. A. Khan and A. Aziz, "Double-diffusive natural convective boundary layer flow in a porous medium saturated with a nanofluid over a vertical plate: prescribed surface heat, solute and nanoparticle fluxes," International Journal of Thermal Sciences, vol. 50, pp. 2154-2160, 2011.

[2] D. A. Nield and A. V. Kuznetsov, "Thermal instability in a porous medium layer saturated by a nanofluid," International Journal of Heat and Mass Transfer, vol. 52, no. 25-26, pp. 5796$5801,2009$.

[3] A. V. Kuznetsov and D. A. Nield, "The onset of doublediffusive nanofluid convection in a layer of a saturated porous medium," Transport in Porous Media, vol. 85, no. 3, pp. 941951, 2010. 
[4] S. U. S. Choi, "Enhancing thermal conductivity of fluids with nanoparticles," in Developments and Applications of Nonnewtonian Flows, D. A. Singer and H. P. Wang, Eds., vol. 231, pp. 99-105, American Society of Mechanical Engineers, New York, NY, USA, 1995.

[5] S. U. S. Choi, Z. G. Zhang, W. Yu, F. E. Lockwood, and E. A. Grulke, "Anomalous thermal conductivity enhancement in nanotube suspensions," Applied Physics Letters, vol. 79, no. 14, pp. 2252-2254, 2001.

[6] T. Hayat and M. Qasim, "Radiation and magnetic field effects on the unsteady mixed convection flow of a second grade fluid over a vertical stretching sheet," International Journal for Numerical Methods in Fluids, vol. 66, no. 7, pp. 820-832, 2011.

[7] W. A. Khan and I. Pop, "Boundary-layer flow of a nanofluid past a stretching sheet," International Journal of Heat and Mass Transfer, vol. 53, no. 11-12, pp. 2477-2483, 2010.

[8] P. Rana and R. Bhargava, "Flow and heat transfer of a nanofluid over a nonlinearly stretching sheet: a numerical study," Communications in Nonlinear Science and Numerical Simulation, vol. 17, no. 1, pp. 212-226, 2012.

[9] T. Hayat, M. Qasim, and S. Mesloub, "MHD flow and heat transfer over permeable stretching sheet with slip conditions," International Journal for Numerical Methods in Fluids, vol. 66, no. 8, pp. 963-975, 2011.

[10] E. Magyari and A. Pantokratoras, "Note on the effect of thermal radiation in the linearized Rosseland approximation on the heat transfer characteristics of various boundary layer flows," International Communications in Heat and Mass Transfer, vol. 38, no. 5, pp. 554-556, 2011.

[11] R. A. Hamid, N. M. Arifin, R. M. Nazar, and I. Pop, "Radiation effects on Marangoni boundary layer flow past a flat plate in nanofluid," in Proceedings of the International MultiConference of Engineers and Computer Scientists 2011 (IMECS '11), pp. 1260-1263, Hong Kong, March 2011.

[12] T. Cebeci and P. Bradshaw, Momentum Transfer in Boundary Layers, Hemisphere Publishing Corporation, New York, NY, USA, 1977.

[13] T. Cebeci and P. Bradshaw, Physical and Computational Aspects of Convective Heat Transfer, Springer, New York, NY, USA, 1988.

[14] A. A. Afify, "Similarity solution in MHD: effects of thermal diffusion and diffusion thermo on free convective heat and mass transfer over a stretching surface considering suction or injection," Communications in Nonlinear Science and Numerical Simulation, vol. 14, no. 5, pp. 2202-2214, 2009.

[15] R. A. Kahar, R. Kandasamy, and Muhaimin, "Scaling group transformation for boundary-layer flow of a nanofluid past a porous vertical stretching surface in the presence of chemical reaction with heat radiation," Computers \& Fluids, vol. 52, pp. 15-21, 2011.

[16] O. D. Makinde and P. O. Olanrewaju, "Buoyancy effects on thermal boundary layer over a vertical plate with a convective surface boundary condition," Journal of Fluids Engineering, vol. 132, Article ID 044502, 4 pages, 2010. 

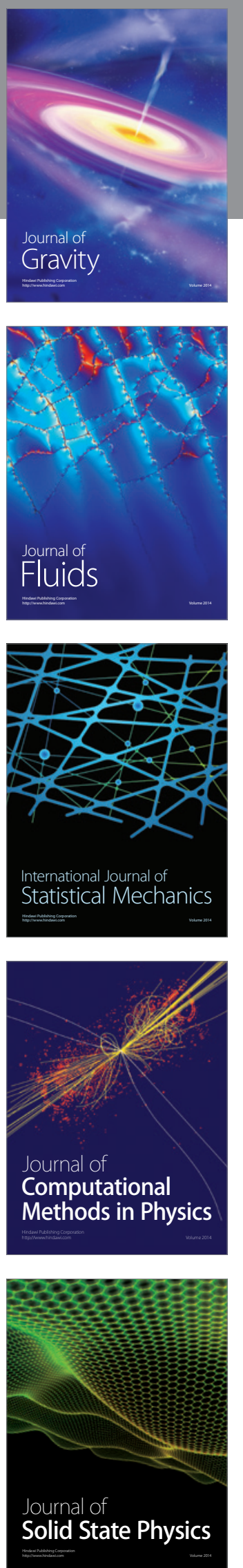
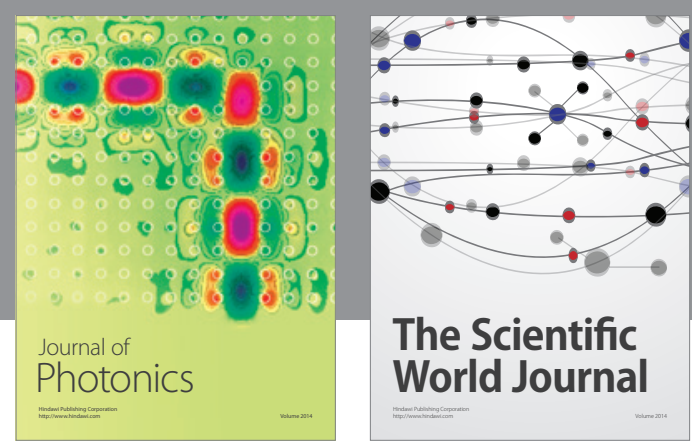

The Scientific World Journal

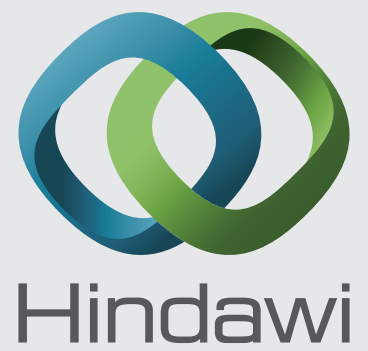

Submit your manuscripts at http://www.hindawi.com
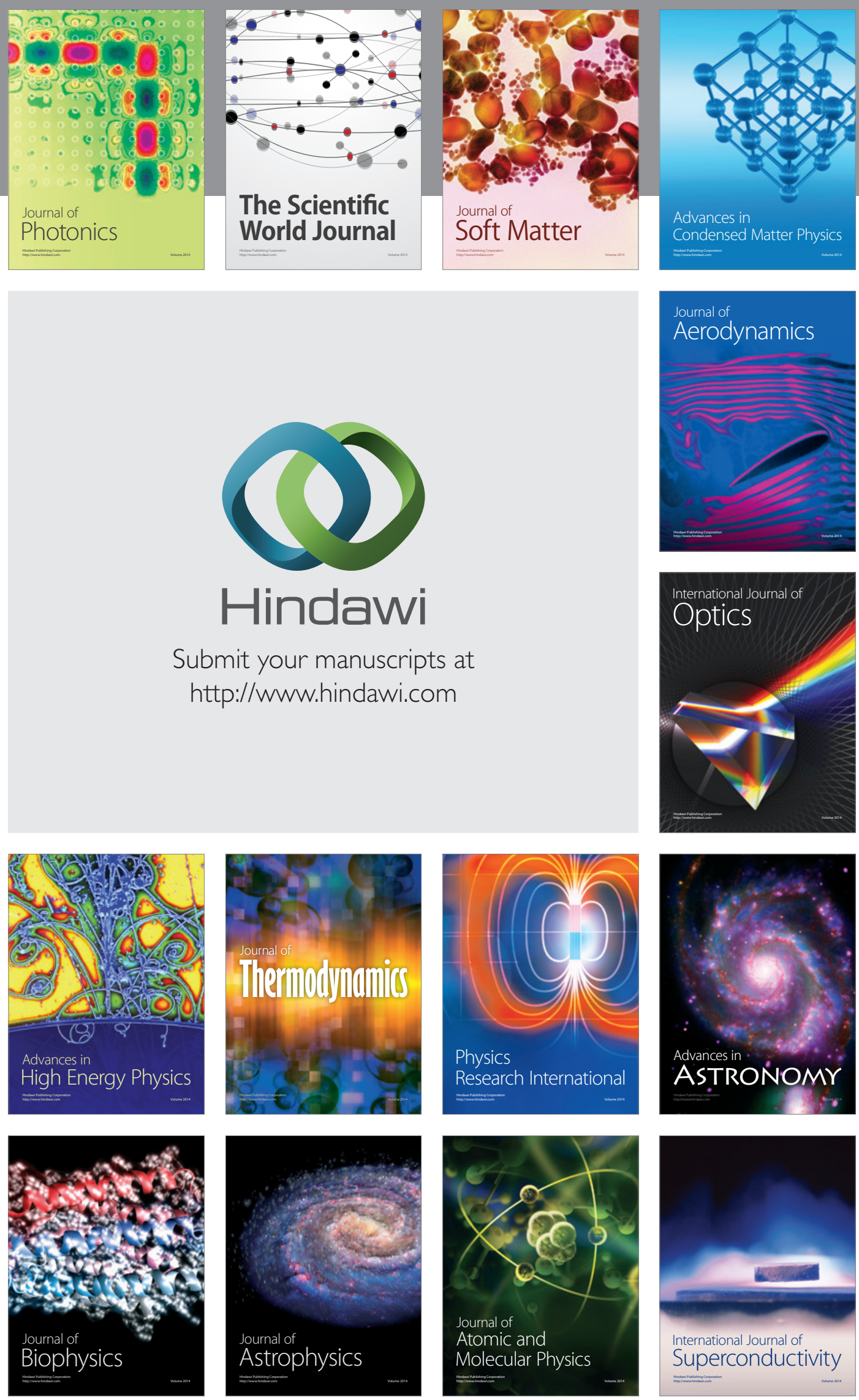
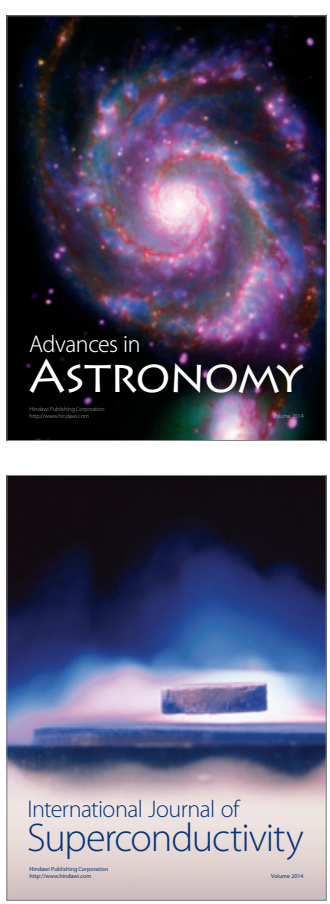